\title{
Otras voces de la locura cantada: usos y significados de la locura en la subcultura punk española (1981-2010)
}

\author{
Other voices of madness in song: uses and meanings of madness in \\ the Spanish punk subculture (1981-2010)
}

Fabián A. Pavez Reyes (https://orcid.org/0000-0003-4439-3048) ${ }^{1}$

Erika Saura Parra (https://orcid.org/0000-0002-1283-9677) ${ }^{2}$

Pedro Marset Campos (https://orcid.org/0000-0001-8510-7111) ${ }^{3}$
${ }^{1}$ The International School of Doctoral Studies, University of Murcia. Edif. Rector Soler, 3․ 30100. Campus de Espinardo, Murcia. Spain. fapavez@uc.cl

${ }^{2}$ Independent Researcher. Murcia Spain

${ }^{3}$ Department of Sociosanitary Sciences, Faculty of Medicine, University of Murcia. Murcia Spain.

\begin{abstract}
The study of depictions of mental disorders in music are scarce, despite the fact that the reproduction of stigmatizing representations can interfere in the search for treatment. The scope of this article is to describe the extent and nature of the descriptions of madness in Spanish punk music (1981-2010) as a basis for comparisons with other countries and musical genres. For the content analysis, 3,653 song lyrics were reviewed looking for the words 'crazy,' 'madness,' or other related terms. A total of 174 works were identified and subsequently analyzed by two decoders. The code book contained 28 codes representing cuatro categories: uses, sense and meanings of 'madness'; main topic of the song; the word 'crazy' in set phrases; and the crazy individual. The use of madness as unpredictability or loss of control was prevalent. In set phrases, links between madness and loss of control were recurrent too. The word 'crazy' was predominant as an attribute or quality. Comparisons with previous findings in the literature suggest that the senses of madness as 'pathology,' 'loss of control,' and 'opposition to reason' were more universal. The identity contents of Spanish punk were the backcloth for the content identified, such that the theme of madness emerges as a sign of identity and a manifestation of otherness.
\end{abstract}

Key words Medicine in the arts, Mental health, Qualitative research
Resumen El estudio de las descripciones de los trastornos mentales en la música es escaso, pese a que la reproducción de visiones estigmatizantes puede afectar la búsqueda de tratamiento. El propósito de este artículo es describir los usos y significados de la locura en el punk español como base para la comparación con otros ámbitos. Análisis de contenido. Se revisó las letras de 3.653 canciones buscando los vocablos "loco", "locura" y otros relacionados. Se identificó 174 obras, analizadas posteriormente por dos codificadores. El manual de codificación abarcó 28 códigos para 4 categorías: usos, sentidos y significados de la locura; tema central de la canción; término "loco" en frases hechas; el loco como personaje. Primaron los usos de la locura como descontrol, desenfreno e impredecibilidad. Entre las frases hechas, también fueron más frecuentes las asociaciones con descontrol. El vocablo loco predominó como expresión de cualidad. La comparación con datos previos sugiere que las ideas de la locura como "patología", "pérdida de control" y "oposición a la razón" tendrían mayor universalidad. Los aspectos identitarios del punk español fueron el telón de fondo para los contenidos identificados, emergiendo la locura como seña de identidad y manifestación de alteridad.

Palabras clave Medicina en las artes, Salud mental, Investigación cualitativa 


\section{Introducción}

La cultura refleja y determina qué se entiende por salud y enfermedad, del mismo modo que los constructos psicopatológicos han sido influidos por los paradigmas científicos y culturales de su tiempo. El conocimiento es integrado al acervo cultural y continúa desarrollándose en interacción con la cultura ${ }^{1,2}$. Así, la relevancia social del estudio de las descripciones de la enfermedad en el arte ha sido defendida previamente ${ }^{3-5}$, considerando el impacto que pueden tener en poblaciones susceptibles las representaciones de ciertas conductas de riesgo (v.g., el consumo de sustancias como el tabaco, alcohol y otras drogas ${ }^{6-11}$ ).

Del mismo modo, la producción y reproducción de representaciones estigmatizantes (tanto de las personas que sufren un trastorno psiquiátrico, como de los tratamientos y los profesionales de la salud mental) tienen un impacto a nivel de salud pública, al dificultar el acceso a la asistencia especializada. Se han descrito representaciones distorsionadas de los profesionales ${ }^{12,13}$, así como el desarrollo y mantenimiento de actitudes negativas hacia las personas que padecen afecciones mentales ${ }^{14,15}$. En este sentido, el estigma y la discriminación figuran como las principales barreras para la búsqueda de tratamiento ${ }^{16,17}$.

Así, la importancia del análisis de medios contiene una doble vertiente. Por una parte, su rol en la comprensión de las ideas circulantes en relación a los trastornos mentales de una población en un período de tiempo dado y, por otra, la identificación del impacto social de dichos contenidos $^{18}$. Respecto al primer punto, la ventaja que ofrece la persistencia en el tiempo de los productos de la cultura popular (documentos sonoros, películas, libros, pinturas, etc.) es el acceso a información cualitativa que no podríamos obtener mediante otras estrategias que por su naturaleza se desarrollan en tiempo presente.

El análisis de las letras de las canciones como prácticas discursivas puede aportar información acerca de las representaciones sociales de la psiquiatría y los trastornos mentales. Para Spink y Frezza $^{19}$, las prácticas discursivas son modos a partir de los cuales las personas componen sentidos y se posicionan en las relaciones sociales de la vida cotidiana. De este modo, como señalan Barros \& Jorge ${ }^{20}$, la investigación en el campo de la cultura constituye un aporte a la comprensión de los sentidos que habitan los imaginarios sociales sobre salud y enfermedad, secundando los esfuerzos emprendidos en el campo de la Salud Pública para consolidar el carácter multidimen- sional del proceso salud/enfermedad, así como poner de manifiesto las relaciones de tal proceso con su contexto socio-histórico ${ }^{20}$.

Por otra parte, la música cantada no sólo refleja las opiniones, sentidos y representaciones de un grupo social, en un momento sociohistórico y un ámbito geográfico dados, sino que también contribuye a la creación de discursos y opiniones sobre los temas retratados. Así, nos enfrentamos a un proceso circular en el que los sentidos, significados y opiniones presentes en la población general nutren y se reflejan en los contenidos de la música popular, al mismo tiempo que son susceptibles de ser metabolizados y devueltos con nuevos significados, los que pueden imponerse o no en los discursos sociales.

El estudio de la música cantada se sustenta en la presencia de un contenido expresivo identificable y susceptible de un análisis sistemático. El análisis de la estructura musical acompañante reviste mayor complejidad, al entrar en juego aspectos proyectivos y altamente subjetivos. Esto puede dificultar la reproducibilidad de los hallazgos, tal como sucede en la interpretación de material pictórico o plástico que, aunque no imposible de analizar, tiene mayores limitaciones que el estudio de la música cantada. Estas consideraciones exigen sacrificar, al menos parcialmente, una parte importante del contenido de las obras analizadas. Parcialmente, porque el componente musical de una canción no queda completamente excluido de un análisis centrado en las letras, dado que provee un contexto para éste, siendo clave para dilucidar intenciones, carga afectiva y otros aspectos de interés.

A pesar de las consideraciones expuestas y el interés artístico por "la locura", el estudio de las representaciones de los trastornos mentales en la música ha sido escaso en la literatura médica ${ }^{18}$. El análisis de contenido ha sido la metodología más frecuentemente utilizada por los trabajos mejor diseñados, dirigidos a temas de interés epidemiológico ${ }^{18}$.

Hasta donde tenemos conocimiento, el único estudio disponible acerca de los sentidos y significados del "loco" en la música popular lo encontramos en Brasil ${ }^{20}$. En este, los sentidos de "locura" identificados fueron: "introspección profunda", "experiencias positivas de perderse en un amor apasionado", "acceso místico a la verdad", "oposición a la razón" y, finalmente, "locura como patología"20. La propuesta de los autores respecto a la posible universalidad de los sentidos identificados en las canciones brasileñas incentiva las comparaciones con los contenidos musicales de 
otras partes del mundo. Así, nos planteamos contribuir a la ampliación de la literatura existente, mediante la descripción de los usos y significados adscritos a la "locura" en la música popular española, a través de la identificación de contenidos y temas asociados. A fin de disminuir la heterogeneidad y maximizar la consistencia interna del análisis, el estudio de subculturas específicas fue necesario. De este modo, nos remitimos al ámbito geográfico español, valiéndonos del ejemplo de un estilo musical concreto, el punk, en un período de tiempo acotado (1981-2010).

Varios factores hacen atractivo el estudio de este género musical. Por una parte, el punk en España tiene una tradición firmemente arraigada y presenta una evolución que puede rastrearse a través de sus exponentes desde principios de los 80 hasta nuestros días, con una influencia reconocida en otras escenas (v.g., Latinoamérica) y en estilos posteriores. Más interesante aún, el origen del punk en España es próximo temporalmente con el advenimiento del Manual Estadístico y Diagnóstico de los Trastornos Mentales (DSMIII, 1979) y corre en paralelo con la reforma psiquiátrica en España (1985) y la evolución posterior de los modelos de atención sanitaria.

Adicionalmente, el punk otorga acceso a contenidos fácilmente identificables, explícitos y crudos, lo que nos aproxima a las expresiones cotidianas y los sentidos de "la gente común" en relación al objeto de estudio. Esto disminuye el sesgo de interpretación, facilitando la concordancia entre codificadores independientes.

El punk como actitud de cuestionamiento a la autoridad y el orden social, ofrece también un rasgo distintivo en cuanto a subcultura que se identifica a sí misma con las personas excluidas socialmente y con "todo aquello que no encaja en una sociedad convencional" 21 . Es indudable que las personas que padecen trastornos mentales, en particular trastornos psicóticos, han sido parte de un colectivo históricamente marginado y un paradigma de "lo que no encaja" en una "sociedad de normales". Por otro lado, el consumo de sustancias, ya sea como seña de identidad de una subcultura, como equivalente de estados mentales alterados o de alienación social, o simplemente como forma maladaptativa de individuación e independencia, incide en el desarrollo de condiciones psiquiátricas comórbidas. Adicionalmente, cobran interés aspectos demográficos (como la vinculación de la música punk con la población adolescente y adulta joven, un período particularmente sensible para el debut de trastornos mentales). Existen descripciones en la literatura de experiencias vitales que relacionan el punk en particular, y el rock en general, con la psiquiatría y los trastornos mentales ${ }^{22,23}$. Del mismo modo, existen datos públicos en España (biografías, entrevistas y documentales) que vinculan la subcultura punk con experiencias personales de trastornos mentales y/o tratamiento psiquiátrico entre sus exponentes, incluyendo trastornos psicóticos, trastornos por uso de sustancias y conducta suicida $^{23}$.

Así, las representaciones de la locura en las canciones punk españolas entre 1981 y 2010 constituyeron el objeto del presente estudio, planteándose como objetivo inicial determinar la extensión y naturaleza de las descripciones de locura. Teniendo esto en mente, nos formulamos las siguientes preguntas de investigación:

¿Cuánto se habla de la locura? (Dimensión cuantitativa: frecuencia de referencias a los vocablos "loco", "locura" y otros equivalentes. Primer nivel de análisis, con énfasis en el texto).

¿Cuáles son los usos, sentidos y significados de la locura que pueden desprenderse de las letras de las canciones? (Dimensión cualitativa: segundo nivel de análisis, inferencial, con énfasis en los temas relacionados y contenidos latentes).

¿Cómo se expresan, con qué temáticas principales se relacionan y cómo esto conecta con la subcultura analizada? (Dimensión cualitativa, predominantemente descriptiva y abierta a futuros trabajos comparativos en búsqueda de elementos de mayor universalidad).

\section{Método}

\section{Marco teórico-metodológico}

El análisis de contenido, en origen, designa una técnica para la descripción objetiva, sistemática y cuantitativa de los contenidos manifiestos de la comunicación ${ }^{24}$. En la actualidad, puede entenderse como un conjunto de métodos y procedimientos de análisis de documentos con énfasis en el sentido del texto ${ }^{25}$. Como consecuencia, establece dos grados de complejidad: descriptivo e inferencial. Así, se busca hacer inferencias a partir de características específicas identificadas de modo objetivo y sistemático en los mensajes ${ }^{26}$. Ruiz Olabuénaga ${ }^{27}$ sostiene que esta lectura sistemática, objetiva, replicable y válida, así como su posterior análisis y teorización pueden efectuarse tanto en el marco y la estrategia metodológica del análisis cuantitativo como del cualitativo. A juicio de Arbeláez \& Onrubia ${ }^{28}$, el análisis de conte- 
nido lo que revela es en definitiva el sentido que emerge del texto.

Así, esta aproximación inductiva se plantea como una estrategia acorde a nuestra pregunta de investigación, toda vez que permite el estudio del sentido de las palabras y el análisis de los temas y categorías propuestas, en ausencia de un marco teórico previo en esta área de estudio.

\section{Muestra}

La población consistió en las letras de las canciones punk españolas publicadas entre 1981 y 2010. Para la selección de material proveniente de grupos representativos del género se recurrió a fuentes documentales, foros web y portales especializados ${ }^{29-34} .177$ bandas punk de todo el territorio español con discos publicados en el período 1981-2010 fueron incluidas y sus discografías revisadas. Las canciones cantadas en un idioma distinto al español, las versiones de otros grupos, los poemas musicalizados y las canciones instrumentales, fueron excluidas del análisis. Las canciones repetidas fueron consideradas sólo una vez (primera versión). Un total de 5.647 canciones cumplieron los criterios de inclusión.

El proceso de recolección y análisis de datos se llevó a cabo en la ciudad de Murcia (España), entre el 11 de septiembre de 2015 y el 7 de diciembre de 2018. A lo largo de este período se efectuó la escucha del material completo, identificándose y transcribiéndose todas aquellas canciones que contuvieron referencias a los términos de interés.

Inicialmente las letras fueron analizadas por dos codificadores independientes en búsqueda de canciones cuya temática principal estuviese relacionada con los trastornos psicóticos o su tratamiento (incluyéndose síntomas psicóticos aislados, alusiones a diagnósticos o términos psiquiátricos, tratamientos psicosociales o biológicos, o vivencias de trastornos psicóticos). Este proceso permitió la identificación de 129 obras. $\mathrm{Al}$ advertir el uso de los vocablos "loco", "locura" u otros equivalentes en más de un cuarto de ellas; se decidió la búsqueda de dichos términos en una muestra aleatoria de 3.653 canciones que no incluyeran a los trastornos psicóticos como tema principal (un $65 \%$ del universo total de canciones). Para el cálculo del tamaño de la muestra se consideró una heterogeneidad del $50 \%$ ante la ausencia de datos previos al respecto, un nivel de confianza del $95 \%$ y un margen de error mínimo $(1 \%)$.

El objetivo del análisis de aquellas canciones cuyas temáticas principales no estuvieron vin- culadas a las psicosis se basó en la necesidad de ampliar los significados adscritos a la locura en la población general, teniendo en cuenta la carga cultural del término, cuyos sentidos exceden el ámbito más concreto de las psicosis. Esta fase llevó a la identificación de 174 canciones adicionales, en las que se focalizó el análisis.

\section{Proceso de codificación y análisis}

La fase cualitativa de la investigación comprendió la selección de frases que contuviesen los términos "loco", "locura", "enloquecer" (y sus declinaciones), "demente", "demencia", así como otros vocablos relacionados de uso coloquial (en adelante, estas frases son designadas como "referencias" y constituyen la unidad de análisis). Aunque cada canción fue considerada como la unidad de contexto principal, éstas podían contener uno o más temas relacionados a la locura. Por estos motivos, se utilizó el concepto de "referencia". Las repeticiones de una misma referencia (v.g., en el coro) no se sumaron a la frecuencia total, considerándose sólo una vez para cada canción.

La codificación se inició en paralelo a la recogida de datos. A diferencia de la investigación puramente cuantitativa, que exige que los datos encajen en códigos preconcebidos estandarizados, la naturaleza de nuestro problema de investigación hizo necesario que los propios datos modelaran los códigos emergentes a lo largo de un proceso recursivo. Como primera aproximación se recurrió a la codificación in vivo para posteriormente dar paso a la organización de los datos en categorías discretas.

En la fase inicial se leyó cuidadosamente los transcriptos sin tomar notas ni considerar una interpretación. Las observaciones generales y lecturas progresivas llevaron al desarrollo de notas marginales con observaciones, palabras clave y nombres asignados para los temas presentes en los datos. Así, los transcriptos fueron revisados y etiquetados para la identificación de aquellos contenidos significativos en la comprensión del problema de investigación planteado. Este proceso permitió separar, compilar y organizar bloques de datos que fueron examinados comparativamente por los investigadores a lo largo del proceso de codificación. Se registró exhaustivamente tantos códigos como fue posible. El proceso se dio por terminado cuando no emergieron nuevos datos novedosos o significativos para el objeto de estudio.

Basados en las descripciones básicas y un proceso de discusión entre los autores, se desarrolló 
un manual de codificación, condensando códigos similares o redundantes y usando un sistema de códigos abiertos que incorporasen nuevos temas en la medida que estos emergieran del texto. Esto fue particularmente relevante en la generación de las categorías "tema principal” y "usos, sentidos y significados". La codificación abierta es defendida por Strauss ${ }^{35}$ y Spradley ${ }^{36}$, quienes apoyan que el sistema de codificación se constituye como una meta de investigación y no sólo como una herramienta para la organización de datos.

El diseño del manual incluyó las dimensiones evaluadas y los códigos que las designaron, así como una guía de los factores a ser tenidos en cuenta por los codificadores para asignar un código particular a cada categoría. Una vez realizado el manual prototipo, se pilotó en un set de canciones. Durante este proceso, se advirtieron las dificultades presentes, tales como categorías/ códigos ambiguos, o datos relevantes no capturados en las categorías desarrolladas inicialmente. Esto guió la realización de cambios en el manual de codificación y un proceso de pilotaje adicional hasta que se consiguió una versión satisfactoria.

A fin de optimizar la consistencia interna, se recurrió a dos codificadores independientes. Los casos de discrepancia fueron resueltos sin dificultad mediante la discusión entre los autores atendiendo en caso necesario a otros datos de contexto para asegurar una correcta interpretación: estructura musical (contexto sonoro), álbum completo en el que estuvo contenida la canción, material impreso, icónico y verbo-icónico.

El manual final de codificación contuvo 28 códigos representando cuatro categorías: "tema principal de la canción" (contexto temático); "usos, sentidos o significados de la locura"; "unidades fraseológicas" (frases hechas); y "el loco como personaje" (Cuadro 1).

El análisis cuantitativo de los contenidos fue de carácter descriptivo mediante medidas de frecuencia.

Al tratarse de un estudio no intervencional basado en datos públicos, no requirió evaluación por Comité de Ética.

\section{Resultados}

Un 2,28\% de las 5.647 canciones evaluadas inicialmente $(\mathrm{N}=129)$ presentaron contenidos relacionados con los trastornos psicóticos o su tratamiento, o bien recurrieron a términos psicopatológicos vinculados a las psicosis. De ellas, un $26,36 \%(\mathrm{~N}=34)$ incluyeron menciones a térmi- nos como "loco", "locura" u otros relacionados. La gran mayoría $(\mathrm{N}=27)$ en un sentido genérico referido a las psicosis; por ejemplo: “Tomás está encerrado en un centro hospitalario. Le acusan de estar loco por su perro imaginario" ${ }^{37}$. En tres casos, el término "locura" fue acuñado como estado mental alterado por efecto del consumo o privación de sustancias. Los cuatro restantes, aludieron a la "locura" como descontrol, pensamiento propio, oposición a la razón o al orden social.

Como se explicitó en el apartado de metodología, el análisis se enfocó en 174 obras que, sin tener a las psicosis como tema principal, incluyeron el uso de vocablos como "loco", "locura" u otros términos relacionados. En este grupo de canciones, las temáticas principales incluyeron visiones críticas del sistema social (35,63\%); amor/desamor, sexo o pasión (12,64\%); aspectos identitarios de la subcultura punk y su estilo de vida $(9,77 \%)$; crimen y violencia $(9,20 \%)$; adversidad social, marginalidad y otros temas sociales $(9,19 \%)$; uso de sustancias $(8,62 \%)$ y temáticas personales $(5,75 \%)$. Un $4,60 \%$ se enmarcó en contextos interdiscursivos (las referencias intertextuales se resumen en el Cuadro 2).

Los vocablos y/o locuciones identificadas fueron: loco, locura, enloquecer, demente, demencia, desquiciado, majara, disfunción mental y mente enajenada. Las palabras "demencia" o "demente" fueron utilizadas como equivalentes de "locura" o "loco" en todos los casos, asociándose a extravagancia o excentricidad, falta de juicio, peligrosidad, conducta violenta o impredecible (en algunos casos, incluso psicopática), a la vez que como adjetivo para situaciones consideradas irracionales o sin sentido (normalmente en un contexto de crítica social). A la inversa, sólo en un caso se utilizó el vocablo "locura" (específicamente, "locura senil") para referirse a demencia.

"Loco" fue el vocablo mayoritario (59\% de las referencias, que aumentó a un 93,26\% al incluir los términos relacionados "locura" y "enloquecer"). En casi un tercio de los casos $(\mathrm{N}=31)$, la palabra "loco" formó parte de frases hechas. $\mathrm{Al}$ analizar dichas unidades fraseológicas encontramos que las asociaciones con desenfreno y descontrol fueron las más recurrentes, con un $83,87 \%$ de las referencias.

"(Estar) loco por" fue una de las frases hechas más frecuentes. Se asoció en la mayoría de los casos a la idea de deseo intenso, relacionándose con temas como: amor y deseo amoroso, avidez y deseo en general, uso de sustancias y hedonismo.

"Loco de atar" y "loco de remate" fueron las 
Cuadro 1. Listado de categorías y códigos incluidos en el manual de codificación.

\begin{tabular}{|c|c|c|}
\hline Categoría & Códigos & Observaciones \\
\hline Tema principal & $\begin{array}{l}\text { "Visiones críticas del sistema social”; "Amor-desamor, } \\
\text { sexo-pasión"; "Identidad punk y estilo de vida"; } \\
\text { "Criminalidad y violencia"; "Adversidad social, } \\
\text { marginalidad y otras temáticas sociales"; "Uso de } \\
\text { sustancias"; "Temáticas personales"; "Intertextualidad"; } \\
\text { "Tópicos misceláneos". }\end{array}$ & $\begin{array}{l}\text { Nueve códigos identificaron los } \\
\text { temas principales de las canciones } \\
\text { con alusiones a los términos "loco", } \\
\text { "locura" u otros relacionados. }\end{array}$ \\
\hline $\begin{array}{l}\text { Usos, sentidos } \\
\text { o significados } \\
\text { de la locura }\end{array}$ & $\begin{array}{l}\text { "Locura como desenfreno, descontrol e } \\
\text { impredecibilidad"; "Locura como privación del juicio } \\
\text { o del uso de la razón"; "Locura como descalificación } \\
\text { o insulto"; "Locura como insensatez"; "Locura como } \\
\text { temeridad"; "Locura como forma de pensar o como } \\
\text { pensamientos / temas de interés propios"; "Locura } \\
\text { como generalidad de estado mental alterado"; "Locura } \\
\text { como caos o agitación"; "Locura como fantasía o idea } \\
\text { disparatada"; "Locura como metáfora de la utopía". }\end{array}$ & \\
\hline $\begin{array}{l}\text { Unidades } \\
\text { fraseológicas }\end{array}$ & $\begin{array}{l}\text { "Estar loco por", "loco de atar", "loco de remate", "loco } \\
\text { suicida", "volver/llevar/traer loco", "tener la cabeza } \\
\text { loca", "hacerse el loco". }\end{array}$ & $\begin{array}{l}\text { Incluye siete códigos relacionados al } \\
\text { uso del vocablo "loco" en el contexto } \\
\text { de frases hechas. }\end{array}$ \\
\hline $\begin{array}{l}\text { El loco como } \\
\text { personaje }\end{array}$ & "Presente" vs "Ausente" & $\begin{array}{l}\text { El código "Presente" da cuenta de las } \\
\text { descripciones del loco como sujeto } \\
\text { participante del relato, más allá del } \\
\text { simple uso del término "loco" como } \\
\text { expresión de cualidad (en dicho } \\
\text { caso, se codificaría "Ausente"). }\end{array}$ \\
\hline
\end{tabular}

Fuente: Elaboración propia.

Cuadro 2. Intertextualidad en canciones punk españolas (1981-2010) que incluyeron los vocablos "loco", "locura" u otros términos relacionados.

\begin{tabular}{|l|l|l|}
\hline \multicolumn{1}{|c|}{ Nombre de la canción (año) } & \multicolumn{1}{c|}{ Grupo musical } & \multicolumn{1}{c|}{ Referencia intertextual } \\
\hline La naranja no es mecánica (1986) & Los Nikis & $\begin{array}{l}\text { La naranja mecánica (cine, 1971; literatura, } \\
1962)\end{array}$ \\
\hline Dorian Grey (1988) & Canívales podridos & El retrato de Dorian Gray (literatura, 1890) \\
\hline Mirando al abismo (1990) & Vómito & Watchmen (cómic, 1986-1987) \\
\hline Kobra Khan (1993) & Los Vegetales & $\begin{array}{l}\text { Masters of the Universe Mini-Comics } \\
\text { (cómic, 1984-1987) }\end{array}$ \\
\hline Harry lo hace por ti (1994) & La Polla Records & Harry el sucio (cine, 1971) \\
\hline Familia de subnormales todos locos (1998) & Airbag & La matanza de Texas (cine, 1974) \\
\hline Mi kompañera la lokura (2003) & Envidia Kotxina & $\begin{array}{l}\text { Don Quijote de la Mancha (literatura, } \\
1605 / 1615)\end{array}$ \\
\hline No es verdad (2007) & El Último ke Zierre & El canto de las sirenas (mitología) \\
\hline
\end{tabular}

Fuente: Elaboración propia.

siguientes en frecuencia. Ambas hicieron referencia a un sujeto "extremadamente loco", aludiendo a la idea de descontrol. Sirva como ejemplo: “Tú que caminas loco de atar, no tienes freno ni autocontrol"38.

"Loco suicida" se utilizó para describir un comportamiento temerario o imprudente, alu- diendo nuevamente a la idea del descontrol: "La ruta 71 , autopista del motor, pista de locos suicidas, infierno de diversión" 39 .

Las frases "volver loco", "llevar loco" o "traer loco" expresaron fascinación en un contexto de atracción física y, en último término, pérdida de control; llegando a la criminalidad en casos ex- 
Cuadro 3. Usos y significados relacionados a la idea de locura como pérdida de control, desenfreno o impredecibilidad.

\begin{tabular}{|l|c|l|}
\hline $\begin{array}{l}\text { Locura como pérdida de control, desenfreno } \\
\text { o impredecibilidad }\end{array}$ & $\begin{array}{l}\text { Número de } \\
\text { referencias }\end{array}$ & \multicolumn{1}{|c|}{ Ejemplos } \\
\hline En general & 37 & $\begin{array}{l}\text { “.. las noches locas, días de infarto" ("El dinero", } \\
\text { El Trono de Judas, 1998) }\end{array}$ \\
\hline Locuciones (frases hechas) & 26 & $\begin{array}{l}\text { Loco de atar; loco de remate; (estar) loco por; } \\
\text { (volver, llevar, traer) loco }\end{array}$ \\
\hline $\begin{array}{l}\text { Locura como equivalente de intoxicación o } \\
\text { privación de sustancias (también como efecto } \\
\text { o resultado de su uso) }\end{array}$ & 5 & $\begin{array}{l}\text { "Vivo bebido y enloquecido, y mi nariz yo la he } \\
\text { perdido" ("Nadie me obliga a cuidarme”, El } \\
\text { Ultimo ke Zierre, 1995) }\end{array}$ \\
\hline $\begin{array}{l}\text { Locura como desenfreno pasional (sexual) } \\
\text { "Es sólo una ilusión, El juego de la pasión. Y me } \\
\text { vuelvo loco sin remedio, loco por tu amor. Loco, } \\
\text { me mola tu cuerpo. Loco, compréndelo" ("Loco", } \\
\text { Cicatriz, 1991) }\end{array}$ \\
\hline
\end{tabular}

Fuente: Elaboración propia.

tremos: “Tengo el pene ensangrentado (...) la maté de un solo golpe (...) La carne, padre, ¡me vuelve loco!" 40 .

En otros casos, el uso figurado "tener la cabeza loca” expresó confusión y embotamiento, introduciendo la idea de locura como disfunción.

Finalmente, "hacerse el loco" aparece como equivalente a "desentenderse", traduciendo desconexión de la realidad: "Y yo me hago el loco. Ignorante animal, siempre con miedo a la miseria..." ${ }^{41}$. Se asemeja a la expresión "hacerse el tonto", que apela al mismo significado; sin embargo, mientras en la primera, "desentenderse" radicaría en la incapacidad del "loco" de conectar con la realidad, en "hacerse el tonto" se alude a la falta de habilidades cognitivas para comprenderla y gestionarla.

En cuanto a la figura del "loco" como personaje, su descripción es minoritaria en este grupo de canciones. En ellas, el vocablo loco se utilizó mayoritariamente para expresar una cualidad, a través de un adjetivo o adjetivo sustantivado, precedido o no de verbos que expresan estado ("estar", "andar" o "ir" loco, "sentirse” loco), cambio ("volverse", "quedarse" o "acabar" loco) o cualidad ("ser" loco). En aquellas obras cuya temática principal se relacionó con los trastornos psicóticos, la figura del "loco" como personaje fue alrededor de cinco veces más frecuente.

Un $6,32 \%$ de las canciones $(\mathrm{N}=11)$ contuvo alusiones puntuales a la locura, impidiendo una mayor profundización en los temas, significados y sentidos (un ejemplo de ello es la canción "En las montañas de la locura" ${ }^{42}$, cuya letra se limita a la repetición de la frase "estás en las montañas de la locura").
En las 163 canciones restantes pudo identificarse más de un sentido en un $11,66 \%$ de los casos. A efectos de presentación de resultados, los vocablos "loco" y "locura" son utilizados indistintamente, englobando en ellos a los otros términos asociados que fueron identificados.

Si bien se contabilizaron 37 referencias generales a la "locura" como desenfreno, descontrol e impredecibilidad, esta frecuencia aumentó a 72 al incluir las unidades fraseológicas identificadas y otros usos que aludieron en sentido último a la pérdida de control (Cuadro 3). Así, la idea del descontrol, el desenfreno y la impredecibilidad llegó a ser una connotación mayoritaria; incluso por sobre el significado de "locura" como privación del juicio o del uso de la razón, acorde a la primera acepción presente en el Diccionario de la lengua española de la Real Academia Españo$\mathrm{la}^{43}$. Adicionalmente, el descontrol y la impredecibilidad aparecieron no sólo como significados adscritos a la locura, sino también como el tema principal de algunas canciones.

En tercer orden de frecuencia se encontró el uso del vocablo "loco" como descalificación o insulto. Otros usos identificados se recogen en el Cuadro 4, incluyendo ejemplos y frecuencias de las distintas dimensiones.

\section{Discusión}

Dentro de los estudios de la música, el área de las representaciones sociales y el estudio de los discursos presentes en la cultura popular ocupa sólo una de las múltiples parcelas existentes. Otros campos de interés son la exposición a contenidos 
Cuadro 4. Usos y significados de los vocablos "loco" y "locura" en 163 canciones punk (España, 1981-2010). El número de referencias no equivale al número de canciones (cada canción puede contener una o más referencias).

\begin{tabular}{|c|c|c|}
\hline Usos y significados & $\begin{array}{l}\text { Número } \\
\text { de } \\
\text { referencias }\end{array}$ & Ejemplos \\
\hline $\begin{array}{l}\text { Locura como pérdida de control, desenfreno o } \\
\text { impredecibilidad }\end{array}$ & 72 & Ver Cuadro 3 \\
\hline $\begin{array}{l}\text { Locura como privación del juicio o del uso de } \\
\text { la razón }\end{array}$ & 54 & $\begin{array}{l}\text { "Ahora dicen que estoy loco, que he perdido la } \\
\text { razón..." ("Esta soziedad", Envidia Kotxina, 2001) }\end{array}$ \\
\hline Loco como descalificación o insulto & 15 & $\begin{array}{l}\text { "Maldito loco, su odio me hace temblar, es un } \\
\text { maestro del crimen y siervo del mal" ("Cobra } \\
\text { Kahn", Los Vegetales, 1996) }\end{array}$ \\
\hline Locura como insensatez o irracionalidad & 10 & $\begin{array}{l}\text { "Mira el fuego que cae del cielo, abrasa y desgarra } \\
\text { niños y madres, sueños y esperanzas. Ilusiones } \\
\text { y sentimientos son ahora alimento de las flores } \\
\text { negras de la locura del hombre" ("Alimento para } \\
\text { las flores", Ruido de rabia, 1987) }\end{array}$ \\
\hline $\begin{array}{l}\text { Loco como persona de poco juicio, } \\
\text { imprudente o temerario }\end{array}$ & 9 & Locuciones relacionadas: "Locos suicidas" $(\mathrm{N}=4)$. \\
\hline $\begin{array}{l}\text { Locura como forma de pensar, pensamiento } \\
\text { propio, aficiones o temas de interés }\end{array}$ & 6 & $\begin{array}{l}\text { "Qué me importa(...) déjame en paz, cada cual } \\
\text { con su locura" ("Escupiré mi ruina", Fe de Ratas, } \\
\text { 2006) }\end{array}$ \\
\hline $\begin{array}{l}\text { Locura como generalidad de estado mental } \\
\text { alterado }\end{array}$ & 5 & $\begin{array}{l}\text { "Si te está acechando la muerte o te han quitado } \\
\text { tu libertad, la locura inunda tu mente" ("No te } \\
\text { puedes rendir", Brote Sikótico, 2007) }\end{array}$ \\
\hline $\begin{array}{l}\text { Locura como caos, actividad frenética, agitada } \\
\text { e intensa }\end{array}$ & 3 & $\begin{array}{l}\text { "Confusión, locura, rabia y agitación, sirenas y } \\
\text { carreras" ("Fuego y cristal”, La Polla Records, 1992) }\end{array}$ \\
\hline Locura como fantasía o idea disparatada & 2 & $\begin{array}{l}\text { "Sólo consigo recordar una locura sobre una } \\
\text { loba, una loba, ¡una loba que vino a por mí!" } \\
\text { ("Licantropía", Tijuana in blue, 1990) }\end{array}$ \\
\hline Locura como metáfora de utopía & 1 & $\begin{array}{l}\text { "He perdido mucha sangre luchando contra } \\
\text { molinos(...) desde el día en que nací rebusqué, } \\
\text { pisoteando hasta en los cubos de basura y } \\
\text { encontré lo mejor, mi compañera la locura" ("Mi } \\
\text { kompañera la lokura", Envidia Kotxina, 2003) }\end{array}$ \\
\hline Otras locuciones & 1 & "Hacerse el loco" \\
\hline
\end{tabular}

Fuente: Elaboración propia.

de riesgo y su potencial impacto en población vulnerable, ya sea a través de aprendizaje vicario, conductas de imitación, o la disminución de la percepción de riesgo, entre otros factores. Este aspecto es relevante en salud pública en relación a ámbitos de la psiquiatría como el uso de sustancias y el suicidio. Sin embargo, la imitación de conductas desadaptativas o la minimización del riesgo percibido respecto del uso de sustancias no son los únicos tópicos de interés cuando tratamos la exposición a contenidos. La producción y reproducción de sentidos estigmatizantes respecto a determinadas condiciones psiquiátricas y su abordaje, pueden tener un impacto en el acceso precoz a los tratamientos, un ámbito de sensible relevancia, por ejemplo, en las fases iniciales de las psicosis.

El interés del arte por la psiquiatría no es algo nuevo. En el punk podemos hallar paralelismos con las visiones de la locura en la ópera romántica y, más directamente, influencias de la contracultura de fines de los 60 y principios de los 70 (como el rock avant-garde), período en el que se han descrito relaciones entre las representaciones de las psicosis en la música popular y el movimiento antipsiquiátrico ${ }^{2,21}$. En líneas generales, tanto en estos estilos musicales como en una parte de la población subsiste un ideal romántico de los trastornos mentales en que se asimila "locura" y creatividad, libertad, sabiduría, ausencia de 
restricciones y oposición al orden establecido. El rock como liberación y ausencia de restricciones, así como su confrontación con el orden social establecido, asume la "locura" como seña de identidad, como la manifestación de su "otredad" * que llega a ser evidente en las formas y contenidos de la música "noise".

Desde el punto de vista musical, el punk puede considerarse inscrito en la tradición del uso del ruido como representación de "locura", a la vez que resistencia o ataque a los moldes musicales socialmente aceptados. Para algunos autores, el ruido aparece como un límite del significado, un reflejo sonoro de un espacio ubicado más allá de la razón, la emulación sonora de la locura ${ }^{44}$. Desde ahí, la así llamada "noise music" constituiría un símbolo de otredad*: el ruido, la locura y el sinsentido en oposición a la música, la razón, el significado y la convención ${ }^{44}$.

Tanto en los aspectos estéticos generales como en los musicales del punk existe una alusión a la locura como escenificación de su automarginación y de la oposición a moldes aceptados socialmente. Mediante un comportamiento y un lenguaje provocador, los punks se autodefinen como execrables, abyectos, "desquiciados", "locos", “caóticos", “enfermos”, “degenerados", e incluso, "esquizofrénicos".

Desde el punto de vista escénico, existen ejemplos en España de las relaciones entre el punk y los trastornos mentales. Es el caso del concierto que el grupo Ataúd Vacante brindó en 1986 en el Hospital Psiquiátrico de Santa Cruz de Tenerife ${ }^{45} \mathrm{o}$ las performances de locura escenificada de Lorenzo Morales, vocalista del grupo "Los Muertos de Cristo"46.

Así, los contenidos hallados y los temas más recurrentes se presentan con el telón de fondo de los aspectos identitarios del punk en España; por lo que futuros estudios comparativos con otros géneros musicales, culturas o países, permitirán comprender qué temas comunes subyacen y presentan mayor universalidad. En la actualidad, el único estudio disponible acerca de los significados del "loco" en la música popular lo encontramos en Brasil ${ }^{20}$. A pesar de las diferencias geográficas, culturales e idiomáticas, existen algunos puntos de convergencia con nuestros hallazgos.

Si bien la cartografía de circulación de sentidos de Barros \& Jorge ${ }^{20}$ describe en una de sus áreas la locura como pasión (fundamentalmente amorosa) y la experiencia positiva de perderse, en nuestros datos encontramos la idea más general de la "pérdida de control", que incluyó no sólo a las experiencias amorosas sino también al uso de tóxicos y a la locura como equivalente de caos. Así, el "perderse" (independiente de la atribución de experiencia positiva o negativa) puede estar en un contexto más amplio de los usos de la locura que el descrito por dichos autores. En cuanto al área de significado de la locura como "patología", encontramos paralelismos en nuestra muestra con aquellos usos que dieron cuenta de la locura como disfunción, psicosis o generalidad de estado mental alterado. El área de "oposición a la razón" también fue identificada en las canciones analizadas, caracterizándose por las alusiones a la temeridad, insensatez e irracionalidad atribuidas a la locura. Dos de las cinco zonas de sentido propuestas por Barros \& Jorge ${ }^{20}$ no fueron relevantes en los usos y significados presentes en las canciones punk españolas. Por una parte, el área de "introspección profunda" o "encuentro consigo mismo", estuvo presente de modo marginal y no constituyó un grupo de significado mayoritario, encontrándose más bien supeditado a otros temas, como por ejemplo la idea de la locura como refugio. En las descripciones y ejemplos mencionados por los autores antes citados (especialmente aquellos pasajes que hacen eco de una visión crítica del funcionamiento social) se intuye cierta proximidad de "la locura como introspección" con una serie de canciones punk cuyos significados se agruparon en torno a la locura como fantasía, pensamiento propio o utopía; aunque pese a ello, no es posible plantear una equivalencia clara. Por otro lado, tampoco encontramos datos consistentes con los sentidos de la locura como "acceso místico a la verdad". Si bien la idea de las relaciones entre locura y sabiduría estuvieron presentes en nuestra muestra, éstas fueron minoritarias y no estuvieron ligadas a aspectos religiosos o metafísicos. De este modo, es posible hipotetizar que ciertos usos y significados pueden ser más universales que otros: la locura como patología, asentada por la tradición médica; la locura como pérdida de control o desenfreno y la locura como oposición a la razón.

En cuanto a los usos y significados preponderantes de la locura en el punk español, fue posible encontrar dos contextos predominantes de significación; por una parte, la desviación de la norma y por otra, el amor, el deseo y la pasión. En estas áreas se inscribieron la mayoría de los usos, destacando la locura como pérdida de control, desenfreno e impredecibilidad y la locura como privación del juicio o del uso de razón.

Por otra parte, la etimología de la palabra loco es un área de amplio debate que excede los objetivos del presente artículo, aunque se hipotetiza 
que puede ser de origen prerrománico. El concepto de locura fue utilizado en Europa con distintos significados según los contextos, pudiéndose corresponder a distintos fenómenos, mal definidos e incluso contradictorios, entendiéndose en general como una desviación de la conducta respecto a la norma. Con el advenimiento de la nosología moderna, el término fue cayendo en desuso hasta llegar a considerarse peyorativo en la actualidad. Sin embargo, su presencia en la cultura popular ha permanecido, dando origen a otros usos que -en buena parte de los casos- se apartan de la idea de "locura" como trastorno mental. Esto explica que la presencia del "loco" como personaje fuera minoritaria en nuestra muestra (más aún al compararla con canciones que tuvieron a las psicosis como tema principal), utilizándose el vocablo fundamentalmente para expresar una cualidad. De las 174 canciones incluidas en nuestra muestra, en casi un tercio de los casos la palabra "loco" se encontró formando parte de locuciones (frases hechas), las que mayoritariamente aludieron al desenfreno y el descontrol. Así, aunque los usos, sentidos y significados de la locura en la cultura popular se aparten de su acepción como entidad nosológica, sí nos orientan a las características atribuidas de modo implícito al "loco".

Desde el punto de vista metodológico, nuestra propuesta es extrapolable a otras manifestaciones artísticas, así como al análisis de medios. Como en todo método de investigación, es necesario tener en cuenta las limitaciones en la generalización de los resultados impuestas por la especificidad del estudio de manifestaciones culturales concretas, en contextos geográficos y temporales determinados. Así, los sesgos ideológicos y culturales presentes en el punk, las diferencias de género (históricamente ha habido una menor representación del género femenino en los estilos musicales derivados del rock) y su carácter subcultural, obligan a ser cautelosos en la generalización de los resultados. Pese a ello, y como se ha señalado previamente, algunos contenidos identificados en nuestra muestra se encuentran también presentes en la música popular brasileña ${ }^{20}$, sugiriendo la presencia de elementos de mayor universalidad. En cuanto a las fortalezas de nuestro estudio se encuentra su originalidad y primacía en el ámbito de la música cantada en español, el tamaño de la muestra analizada, y la propuesta de una estrategia replicable que facilita la comparación con futuros trabajos de naturaleza similar que puedan llevarse a cabo en torno a otros géneros musicales o ámbitos geográficos.

En síntesis, el análisis de medios y de los productos culturales puede ser una fuente de conocimiento adicional que nos conecte con las representaciones sociales de nuestra profesión y su ámbito de acción, favoreciendo una mejor comprensión de lo que entienden nuestros pacientes y la población en general por "psiquiatría" o "trastorno mental". Adicionalmente, las descripciones de los trastornos mentales en el arte constituyen un recurso para la educación de los usuarios, sus familias, estudiantes de medicina y médicos no especialistas. De manera transversal, el análisis a través del arte de las manifestaciones subculturales ligadas a los padecimientos mentales puede dar algunas pistas para el trabajo con subculturas específicas y acercarnos a los sentidos, significados y usos (cada vez más frecuentes) de los términos psicopatológicos en la cultura popular.
* Otredad: Noción habitual en filosofía, antropología, sociología y otras ciencias sociales. Se relaciona a la asunción de identidad basada en el reconocimiento del otro como diferente. A nivel social, suele derivar en la creación de grupos subordinados; así, unos encarnarán la norma y la identidad valorada y otros serán definidos por sus faltas, devaluados y susceptibles de discriminación $^{47}$. 


\section{Colaboradores}

F Pavez-Reyes fue responsable de la concepción y diseño del estudio. Ejecución y análisis (selección de material y revisor una análisis de contenido). Interpretación de datos y preparación del manuscrito (redacción y correcciones, aprobación de la versión final). E Saura, ejecución y análisis (segundo revisor independiente análisis de contenido, discusión de resultados). P Marset, revisión y aprobación del proyecto. Preparación del manuscrito (comentarios para la corrección, aprobación de la versión final).

\section{Referencias}

1. Erfurth A, Hoff P. Mad scenes in early 19th-century opera. Acta Psychiatr Scand 2000; 102(4):310-313.

2. Spelman N. All the madmen: Popular music, anti-psychiatry and the myths of madness [thesis]. Salford: School of Media, Music and Performance; 2009.

3. Baxendale $S$. The representation of epilepsy in popular music. Epilepsy Behav 2008; 12:165-169.

4. Feggetter G. Suicide in opera. Br J Psychiatry 1980; 136(6):552-557.

5. Oksanen A. Addiction and rehabilitation in autobiographical books by rock artists, 1974-2010. Drug Alcohol Rev 2013; 32(1):53-59.

6. Primack B, Dalton M, Carroll M, Agarwal A, Fine M. Content analysis of tobacco, alcohol, and other drugs in popular music. Arch Pediatr Adolesc Med 2008; 162(2):169-175.

7. Primack B, Nuzzo E, Rice K, Sargent J. Alcohol Brand Appearances in U.S. Popular Music. Addiction 2012; 107(3):557-566.

8. Christenson P, Roberts D, Bjork N. Booze, drugs, and pop music: trends in substance portrayals in the Billboard top 100: 1968-2008. Subst Use Misuse 2012; 47(2):121-129.

9. Herd D. Changes in the prevalence of alcohol in rap music lyrics 1979-2009. Subst Use Misuse 2014; 49(3):333-342.

10. Hardcastle K, Hughes K, Sharples O, Bellis M. Trends in alcohol portrayal in popular music: A longitudinal analysis of the UK charts. Psychol Music 2015; 43(3):321-332.

11. Holody K, Anderson C, Craig C, Flynn M. “ Drunk in Love ": The Portrayal of Risk Behavior in Music Lyrics. J Health Commun 2016; 21(10):1098-1106.

12. Bischoff R, Reiter A. The role of gender in the presentation of mental health clinicians in the movies: Implications for clinical practice. Psychother Theor Res Pract Train 1999; 36(2):180-189.

13. Gabbard G, Gabbard K. Cinematic stereotypes contributing to the stigmatization of psychiatrists. In: Fink PJ, Tasman A, editors. Stigma and Mental Illness. Washington: American Psychiatric Press; 1992.p. 113127.

14. Granello D, Pauley P. Television viewing habits and their relationship to tolerance toward people with mental illness. J Ment Heal Couns 2000; 22(2):162175.

15. Reda S. Public perceptions of former psychiatric patients in England. Psychiatr Serv 1996; 47(11):12531255.

16. Satcher D. Executive summary: a report of the Surgeon General on mental health. Public Health Rep 2000; 115(1):89-101.

17. Brown K, Bradley L. Reducing the stigma of mental illness. J Ment Heal Couns. 2002; 24(1):81-87.

18. Pavez F, Saura E, Perez G, Marset P. Social Representations of Psychiatry and Mental Disorders Examined Through the Analysis of Music as a Cultural Product. Music Med 2017; 9(4):247-253.

19. Spink M, Frezza R. Práticas Discursivas e produção de sentidos: a perspectiva da Psicologia Social. In: Spink MJ, organizer. Práticas discursivas e produção de sentidos no cotidiano: aproximações teóricas e metodológicas. Sao Paulo: Cortez; 2004. p. 17-39. 
20. Barros JPP, Jorge MSB. Vozes da Loucura Cantada: Sentidos sobre a Loucura e o Louco em Canções Brasileiras. Cien Saude Colet 2011; 16:4845-4855.

21. Gios TS, Lotufo Neto F. Termos psicopatológicos em bandas e músicas de rock and roll. J Bras Psiquiatr 2013; 62(1):46-50.

22. McDonald J. Psychiatry rocks. Br J Psychiatry 2012; 200(6):453.

23. Pavez F. Portrayals of Psychiatry and Mental Disorders in Hispanic Rock Music. Acad Psychiatry 2017; 41(6):741-749.

24. Berelson B. Content Analysis in Communication Research. NY: Free Press; 1952.

25. Amezcua M, Gálvez A. Los modos de análisis en investigación cualitativa en salud: perspectiva crítica y reflexiones en voz alta. Rev Esp Salud Publica 2002; 76(5):423-436.

26. Bryman A. Social Research Methods. Oxford: Oxford University Press; 2015.

27. Ruiz-Olabuénaga J. Metodología de la Investigación Cualitativa. Bilbao: Universidad de Deusto; 2012.

28. Arbeláez M, Onrubia, J. Análisis bibliométrico y de Contenido: Dos metodologías complementarias para el análisis de la Revista Colombiana Educación y Cultura. Revista de Investigaciones UCM 2014; 14(23):1431.

29. Diccionario de punk y hardcore: España y Latinoamérica. Madrid: Fundación Autor; 2011.

30. Alfonso J. Hasta el final: 20 años de punk en España. Madrid: Zona de Obras; 2002.

31. Alfonso J, Bocos A. No Acepto!!! 1980-1990: Diez Años de Hardcore, Punk, Ira y Caos [DVD]. Spain; 2007.

32. La Fonoteca [Internet]. 2019 [cited 2019 Feb 5]. Available from: http://lafonoteca.net/grupos/.

33. MusicaPunk.net [Internet]. 2013 [cited 2019 Feb 5]. Available from: http://musicapunk.net/.

34. Maneras de vivir [Internet]. 2018 [cited 2019 Feb 5]. Available from: http://www.manerasdevivir.com/ foro/

35. Strauss A. Qualitative Analysis. N.Y.: Cambridge University Press; 1987.

36. Spradley J. Participant Observation. N.Y.: Rinehart \& Winston; 1980.

37. Trastienda RC. Tomás. In Revolución [CD]. Madrid: Working Class Records; 2006.

38. El Último ke Zierre. Cementerio de Lobos. In Ay de mí! [CD]. Valencia: Maldito Records; 2003.
39. Delincuencia Sonora. Ruta 71. In Por la causa de algún rebelde [LP]. San Sebastián: Basati Diskak; 1990.

40. El Último ke Zierre. La Carne. In Que se repartan el mundo [CD]. Algorta: Discos Suicidas; 1993.

41. El Último ke Zierre. Me hago el loco. In Que se repartan el mundo [CD]. Algorta: Discos Suicidas; 1993.

42. Vómito. En las montañas de la locura. In A un paso de la locura [LP]. Algorta: Discos Suicidas; 1990.

43. Real Academia Española. Locura [Internet]. Diccionario de la lengua española; 2014. Available from: http:// dle.rae.es/srv/fetch?id=NYYDU9d

44. Thompson M. Releasing the Inner Idiot: Noise Music, Marginality and Madness. In: Wolf M, Hill A, editors. Proceedings of Sound, Sight, Space and Play. UK: University of Lincoln; 2010. p. 1-11.

45. Motrocos. Ataúd Vacante Hospital Psiquiátrico Tenerife. [video file]. 2017 June 4. [cited 2018 Sept 11]. Available from: http://www.youtube.com/watch?v=xi_ZL pRCijc.

46. SiVis Pacem. Los Muertos De Cristo Ladran, Luego Cabalgamos DVD-01. [video file]. 2013 March 9. [cited 2018 Sept 11]. Available from: http://youtu.be/ Y6whxb9Wetk?t=38m47s.

47. Staszak J. Other/Otherness. In: Kitchin R, Thrift N, editors. International Encyclopedia of Human Geography. Oxford: Elsevier; 2009. p. 43-47.

Artículo presentado en 20/02/2019

Aprobado en 29/07/2019

Versión final presentada en 01/08/2019

Editores jefes: Romeu Gomes, Antônio Augusto Moura da Silva 